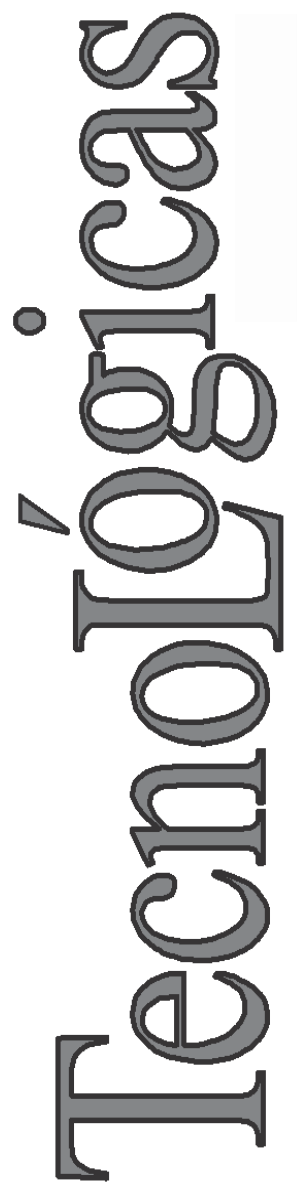

\title{
Gestión de Comunicaciones en los Proyectos
}

\section{Project Communications Management}

José C. Santiago-Guevara ${ }^{1}$

Mauricio Rojas-Contreras ${ }^{2}$

Luis A. Esteban-Villamizar ${ }^{3}$

1 Universidad de Pamplona, Pamplona-Colombia josesantiago@unipamplona.edu.co

2 Universidad de Pamplona, Pamplona-Colombia mrojas@unipamplona.edu.co

3 Universidad de Pamplona, Pamplona-Colombia lesteban@unipamplona.edu.co 


\title{
Resumen
}

Este artículo presenta una revisión con relación al objeto de estudio: Gestión de Comunicaciones como factor importante en la gestión de proyectos. Dicha revisión incluye los artículos más relevantes y los autores más reconocidos en el campo de la gestión de proyectos, haciendo énfasis en los proyectos de telecomunicaciones, lo que permite definir un modelo de gestión de comunicaciones.

\section{Palabras clave}

Gestión de la comunicación; proyectos; telecomunicaciones; evolución histórica; procesos.

\begin{abstract}
This article presents a review in relation to the object of study: Communications Management as an important factor in the management of projects. The review includes the most relevant and most renowned authors in the field of project management, focusing on telecommunications projects, which lets you define a communications management model.
\end{abstract}

\section{Keywords}

Communication management; project; telecommunication; historical evolution; processes. 


\section{INTRODUCCIÓN}

La guía $\mathrm{PMBOK}{ }^{\circledR}$ define que la gestión comunicaciones dentro de proyecto, incluye los procesos para garantizar que la generación, la recopilación, la distribución, el almacenamiento, la recuperación y la disposición final de la información del proyecto sean adecuados y oportunos (PMBOK, 2008). Esta concepción también se puede observar en otros estándares, guías y metodologías para la gestión de proyectos como lo son PRINCE2 y la ISO21500. Este artículo es producto de parcial de un proyecto de investigación, cuyo objetivo principal pretende obtener y validar un modelo de gestión de comunicaciones para los proyectos de telecomunicaciones.

El estudio y aplicación de la gestión en las comunicaciones ha tomado importancia en los últimos años como lo confirma (Rivera, B., et al., 2005) al afirmar que la comunicación desde todas las perspectivas de estudio (organizacional, comercial, interpersonal), conforman un conjunto de instrumentos, estrategias y acciones importantes en todos los proyectos. Autores como (Scheinsohn, D., 1993) plantean su posición determinante al manifestar que "la comunicación es un hecho fundante de las relaciones en las organizaciones". De alguna manera esta afirmación manifiesta que la comunicación propicia una sana relación entre los miembros del equipo. (Fernández, C., 1991) considera que la comunicación es el sistema nervioso de la organización. (Rivera, B., et al., 2005) a partir del interrogante: ¿Es importante la Comunicación para el funcionamiento de las organizaciones?, llega a la conclusión de que es un factor clave de éxito en todo proyecto.

\section{ANTECEDENTES}

En la comunicación se considera que las teorías fundadoras son las ideas de Aristóteles (Quintín, 1990), a partir de la concepción de la comunicación como la capacidad para gestionar mensajes y significados (Pérez, A., 2006). Contextualizando la gestión de comunicaciones a los proyectos de telecomunicaciones, se puede afirmar que antes de los años 50' no existía la gestión de comunicaciones como un proceso formal en los proyectos, por ello 
se hace un recorrido por los proyectos de telecomunicaciones a través de la historia hasta la mitad del siglo XX, con la aparición de la telegrafía óptica se construyeron las primeras redes de telecomunicación propiamente dichas. Datan de la revolución francesa, cuando Claude Chappe, ideó una red óptica-mecánica en 1792 (Estepa, R., 2004). Con los descubrimientos de Ampere y Faraday se construyó el telégrafo eléctrico. El estadounidense Samuel Morse llevó a cabo la primera transmisión telegráfica entre Washington y Baltimore, en 1844. En 1866 se instaló el primer cable trasatlántico que unía América con Europa. Alexander Graham Bell en febrero de 1876, registra la patente del teléfono.

En 1877 nace la empresa Bell (Estepa, R., 2004). En 1878 aparece el primer tablero de conmutación manual con capacidad para 21 abonados y en 1892 la primer central telefónica automática e instalada en Indiana EE.UU gracias al señor Almon B.Strowger (Joskowicz, J., 2012). En 1901, Marconi hace el primer enlace transatlántico utilizando ondas de radio. En 1915 se inaugura la línea telefónica más larga de la época entre New York y San Francisco. En 1927 el francés Alec Reeves crea la Modulación por Pulsos Codificados PCM. En la década de los años 40’ empieza la era de las computadoras. Finalizando la mitad de siglo, en 1946 AT\&T presenta el primer sistema de telefonía móvil vehicular y en 1948 se da vida al transistor que a su vez optimizaría sustancialmente los sistemas de telecomunicaciones (Joskowicz, J., 2012).

\section{LA GESTIÓN DE COMUNICACIONES EN PROYECTOS}

Después de la Segunda Guerra Mundial tiene sus orígenes la Gestión de Proyectos como disciplina de estudio y se le reconoció como herramienta necesaria para la realización de actividades tecnológicas complejas de manera interrelacionada y coordinada, haciéndole eficiente el manejo de los recursos (Butler, A., 1990; Gyepro, 2005; Conalep, 2013). Se afirma además que la gestión de proyectos surgió como resultado de la complejidad de los problemas gubernamentales, militares y comerciales (Gyepro, 
2005). En los años 60' se desarrollaron herramientas que favorecieron el surgimiento de la Gestión de Proyectos como el PERT, GANTT y el CPM (Gyepro, 2005). El arquitecto de desarrollo de misiles balísticos Polaris Bernard Schriever, es considerado el padre de la gestión de proyectos, Bernard desarrolló el concepto de "concurrencia" integrando todos los elementos del proyecto en un solo programa y presupuesto, ejecutándolos al tiempo y no por fases. Con ello logró reducir sustancialmente los tiempos de ejecución (Palacio, J., 2006). Posteriormente surgieron técnicas específicas, histogramas, cronogramas, los conceptos de ciclo de vida del proyecto o descomposición en tareas WBS. (Palacio, J., 2006).

Los proyectos de la época requerían del trabajo conjunto y sincronizado de varias disciplinas e hizo evidente en los años 60' la necesidad de desarrollar métodos de organización y trabajo. Como respuesta, se crearon algunas organizaciones las cuales desarrollaron el cuerpo del conocimiento de la gestión de proyectos, en 1965 es fundado el IPMA (Internacional Project Managenet Association), posteriormente en el año1969 se creó el PMI (Project Management Institute) y más tarde en 1989 surgió PRINCE2 (PRojects IN Controlled Environments). Estas organizaciones se han convertido en la guía de la profesión denominada gestión de proyectos.

De igual manera la gestión de las comunicaciones ha estado presente en dichas guías, metodologías, estándares o cuerpo del conocimiento como un área de relevante importancia. Para (Ramírez, F., y Rojas, L., 2004) la gestión de comunicación es un campo de la práctica fundamentada en forma reflexiva $\mathrm{y}$ articulada que se extiende a través de un gran número de niveles de la actividad comunicacional en la organización. En las décadas posteriores a los años 60', la gestión de proyectos se basó en la planificación y en el seguimiento y control. Esta planificación se basa en un análisis detallado del trabajo y su división en tareas, que parte de unos requisitos iniciales de lo que se quiere realizar. Para (Preciado, A., y Ramírez, H., 2012) si una organización cuenta con una buena estrategia de comunicación, también cuenta con un plan de gestión. Por ello la gestión de la comunicación y el plan de gestión suelen ser sinónimos (Pizzolante, I., 2004; Petit, 
A., 2004; Manuci, 2006). Los estudios realizados sobre comunicación en las organizaciones por (Grunig, E., 1992, 2000; Sotelo, C., 2001; Cornelissen, J., 2008), concluyen que la comunicación permite dar cumplimiento a la necesidad sentida de la organización, de ajustarse a los contextos sociales (Garrido, J., 2003). De igual forma (Gibson, J., 1999) plantea que si una organización quiere obtener éxito en sus comunicaciones debe implementar un flujo bidireccional a través del uso de las TIC (Tecnologías de la Información y las Comunicaciones).

Partiendo del axioma: "tanto si se lo propone o no, la organización siempre comunica” (Watzlawick, P., 1981), la gestión estratégica de la comunicación se transforma en un vector de competitividad (Álvarez, A., 2011). Para (Goldhaber, G., 1984) la comunicación en una organización es un flujo ascendente, descendente y horizontal. (Rojas, L., y Arape, E., 1999) refuerzan este planteamiento al afirmar que la comunicación es un intercambio progresivo que logra el crecimiento común de todos los miembros de la organización. Con relación a ello (Senge, P., 1995) infiere que toda organización debe estar en capacidad de mantener sus propias comunicaciones. Para (Timm, P., 1986) la comunicación es esencial en la organización con relación a obtener cooperación y consenso. Basados en la planificación del trabajo se estiman los recursos y tiempos disponibles, y durante la construcción se sigue de cerca la ejecución para detectar posibles desviaciones y tomar medidas para corregirlas (Palacio, J., 2006). Se trata por tanto de conseguir que el desarrollo del proyecto se lleve a cabo según lo planeado. Dentro ello es de mucho valor el aporte que hace al proyecto una adecuada gestión de comunicaciones. Tradicionalmente la gestión de proyectos implica el trabajo hecho dentro del alcance, tiempo y presupuesto planificados, variables conocidas como "el triángulo de prioridades o de hierro" (Archibald, R., 2000).

\subsection{Evolución Histórica de Metodologías de Gestión de Proyectos}

\subsubsection{Gerentes de Proyectos en la Antigïedad}

Se destacan proyectos como el realizado 2550 A.C. cuando el hombre construyó una pirámide que requiere de 20 años de 
trabajo. El emperador Qin Shi Huang 206 A.C, en la construcción de la Gran Muralla China organizó la fuerza de trabajo al ordenar que se ubicaran en tres grupos: soldados, civiles y criminales. Para el año 1750 los líderes empresariales buscaron equilibrar la gestión de mano de obra y la fabricación mecanizada a través de lo que se denominó la revolución industrial. En EE.UU. el ferrocarril transcontinental creado en 1896 requirió gran gestión de recursos y actividades.

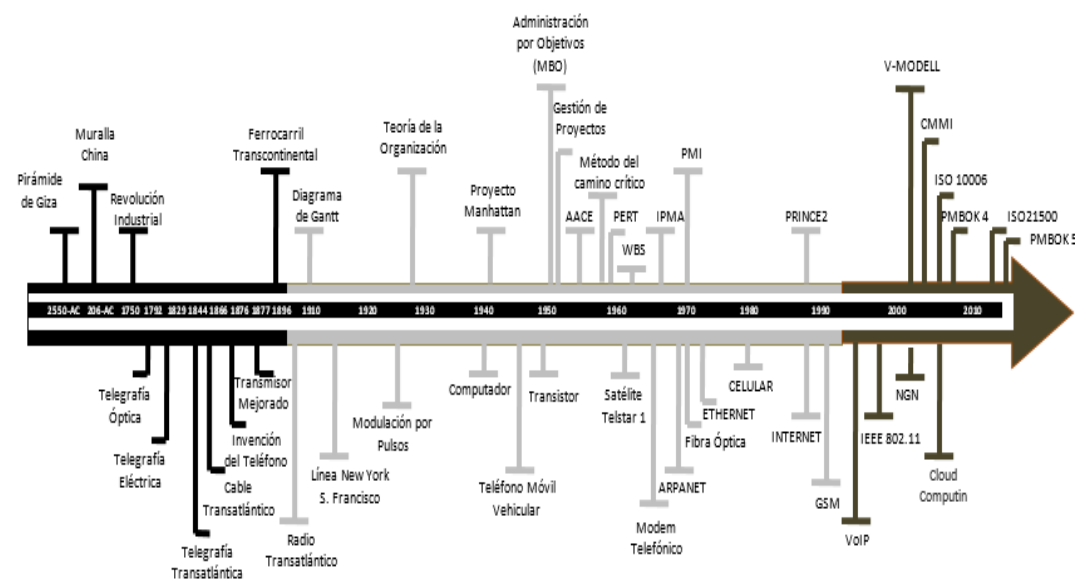

Fig. 1. Evolución histórica de los proyectos y sus herramientas. Fuente: Autores

\subsubsection{Gestión de Proyectos en el Siglo XX y XXI}

En 1912 el Ingeniero Henry Gantt desarrolla el "Diagrama de Gantt", éste fue utilizado por primera vez en la Primera Guerra Mundial para ayudar a construir barcos. Para el año 1937, se escribe el primer documento sobre la teoría de la organización. Manhattan Engineering District (MED) desarrollo el Proyecto Manhattan durante la Segunda Guerra Mundial para crear las primeras armas nucleares en el año de 1945. El General Bernard Schrieve de la Fuerza Aérea de EE.UU. acuña el término "Gestión de Proyectos" a través de su experiencia en la fabricación de misiles. En ese mismo año, el austriaco Peter Drucker desarrolla las guías prácticas para la administración por objetivos (MBO). Asociación Americana de Ingenieros de Costos (AACE) es creada en el año 1956. DuPont y Remington Rand diseñaron en 1957 el 
Método del Camino Crítico. En 1958 es desarrollado y utilizado por la Marina de los EE.UU. el PERT, a través del programa de misiles Polaris. El Departamento de Defensa de EE.UU. en 1962 crea el método para dividir proyectos en componentes más pequeños (WBS). En 1965 se creó el IPMA, organización suiza para la gestión de proyectos y en 1969 se crea el PMI. Estos organismos promueven desde entonces hasta el día de hoy la gestión de proyectos como disciplina y profesión. Por último en 1989 se desarrolla la Metodología de Dirección de Proyectos para un Entorno Controlado PRINCE2. En el año 2000 se crea el Método de gestión de proyectos V-MODELL. CMMI (Capability Maturity Model Integration), para procesos en empresas de TI es desarrollado en el año 2002. Posteriormente es publicado en año 2003 la norma ISO10006 para la Gestión de Calidad en Proyectos. El siguiente año se publica la Guía PMBOK versión 3. En el año 2008 se publica la Guía PMBOK versión 4. En el año 2012 se publica la norma ISO21500 para la Gestión de Proyectos. A inicios del año 2013 se publica el PMBOK5.

\subsection{Los Proyectos en Telecomunicaciones}

En la sección antecedentes de este artículo se abordaron los desarrollos más importantes surgidos antes de los años 50'. En la segunda mitad del siglo XX a la par de la gestión de proyectos, aparecen otros adelantos en el campo de las telecomunicaciones como la instalación del primer cable telefónico transatlántico en 1956. En 1962 es lanzado el primer satélite de comunicaciones Telstar 1 y en ese mismo año es instalado el primer sistema de trasmisión digital T1. En 1966 AT\&T desarrolla el primer modem telefónico y en 1969 se crea la primera red de computadoras Arpanet. En el año 1970 se fabrican las primeras fibras ópticas y en 1973 Bob Metcalfe crea la tecnología Ethernet. Para ese mismo año aparecen los primeros teléfonos celulares. Más tarde en 1988 se instala el primer cable transatlántico de fibra óptica entre EE.UU y Francia. En 1989 nace la Internet de la mano de Tim Barners Lee en el Instituto Europeo de Investigación de Física de partículas (CERN). 
En 1991 se crea la tecnología celular GSM y 1996 aparece la telefonía sobre protocolo IP. En 1997 se aprueba el estándar IEEE 802.11 para las redes inalámbricas. En 2005 se lanza al mercado VDSL2. En 2009 se crea el estándar IEEE 802.11n. En los últimos años se centra la investigación en los conceptos y aplicación de Redes de Nueva Generación NGN, servicios en la nube, entre otros. Las innovaciones continuas en las (TIC) han transformado las economías inducidas por las decisiones de los agentes del mercado en cualquier parte del mundo (Freeman, C., y Louca, F., 2001). Con relación a ello la gestión de proyectos no ha sido ajena al proceso de evolución de las telecomunicaciones, por el contrario ha participado de manera activa en el proceso de transformación. Dentro de la gestión de proyectos emergen áreas del conocimiento puntuales como la ya mencionada gestión de comunicaciones, pero ¿qué hace particular la gestión de comunicaciones en los proyectos de telecomunicaciones? La repuesta se basa en características de forma y no de fondo, ya que la gestión de comunicaciones está establecida bajo procesos genéricos que permiten su aplicación en cualquier tipo de proyecto sin importar la disciplina, por ello su particularidad se establece en el tipo de información que se genera.

\section{CONTEXTO REGIONAL}

En los proyectos de telecomunicaciones es difícil encontrar con claridad un plan, modelo o documento que formalice su Gestión. Para (Srivannaboon, S., 2005) algunas organizaciones ni siquiera conocen la gestión de proyectos. En una investigación realizada por (Rivera, B., et al., 2005; Clemenza, C., et al, 2002), para la Universidad del Zulia Venezuela, concluyen que la alta gerencia tiene limitaciones para llevar a la práctica la difusión de la política comunicacional y no satisfacen los requerimientos de la comunidad. (Prieto y Suárez, 1998) resaltan que una comunicación asertiva entre la alta gerencia universitaria y la comunidad en general, permite un mejor desempeño en las tareas. La alta gerencia debe ser la encargada de propender por su gestión (Serna, H., 2000). Por su parte (Riel, 1997) manifiesta que la comunicación 
debe alcanzar todos los niveles de la organización. De igual manera (Vargas, L., 2003) considera que la comunicación debe favorecer el cumplimiento de los objetivos.

En la tesis: "La gestión de los proyectos en las empresas del sector energético. Caso: Enelven - Carbozulia”, el autor concluye que no se desarrolla un plan comunicaciones (Ugas, L., 2008). Para (Arce, L., y López, S., 2010) el 53\% de las empresas Bogotanas encuestadas en su estudio "Valoración de la gestión de proyectos en empresas de Bogotá”, no cumplen con la aplicación de las buenas prácticas en gestión de proyectos. En el estudio de Caso: Comunicación en las Organizaciones y TIC, (Morales, R., y González, V., 2010) concluye que en el área de la docencia de la educación superior existen falencias en la gestión de comunicación organizacional. En otro estudio realizado en la ciudad de Bogotá por (Bustos, M., et al, 2010) denominado "Un caso de gestión de la comunicación y la información en la pyme manufacturera de calzado chiquitines" hallaron que al revisar los resultados, el factor de la comunicación presentaba niveles bajos de gestión.

\section{MODELO PARA GESTIÓN DE COMUNICACIONES PERH}

Se le denomina modelo PERH por sus componentes de: Procesos, Estrategias, Roles y Herramientas. Planteado para la gestión de comunicaciones en proyectos de telecomunicaciones. Este modelo es el resultado del análisis de la información obtenida a través de la presente revisión y la aplicación de varios instrumentos de consulta como encuestas y entrevistas a expertos de área de proyectos en telecomunicaciones. Está estructurado de manera que puede ser ajustado a cualquier guía, modelo o norma de gestión de proyectos. De igual forma el modelo se comporta de manera cíclica, ello indica que será actualizado y desarrollado en el tiempo a través de las lecciones aprendidas.

- Componente de Procesos: se definen los mecanismos a tener en cuenta de acuerdo a las características del proyecto con el objetivo de generar la Matriz de Comunicaciones. 
- Componente de Estrategias: aborda y da importancia a la toma de decisiones prioritarias que surgen del análisis que define la tarea y el modo de cumplirla, utilizando herramientas de comunicación adecuadas.

- Componente de Roles: los roles dentro de los proyectos de telecomunicaciones se han de clasificar en tres categorías: los Roles Estratégicos, los Roles Misionales y los Roles de Soporte. Esta clasificación se hace teniendo en cuenta las diferentes actividades y grados de participación por parte de los interesados en el proyecto a lo largo de su vigencia.

- Componente de Herramientas: de acuerdo a las herramientas, el modelo PERH determina los procesos para el desarrollo de estrategias de comunicación dentro del proyecto de telecomunicaciones. Cabe resaltar que partiendo de los resultados de la aplicación de cada herramienta, el gestor del proyecto y sus colaboradores tienen una información de primera mano para la toma de decisiones dentro del proyecto.

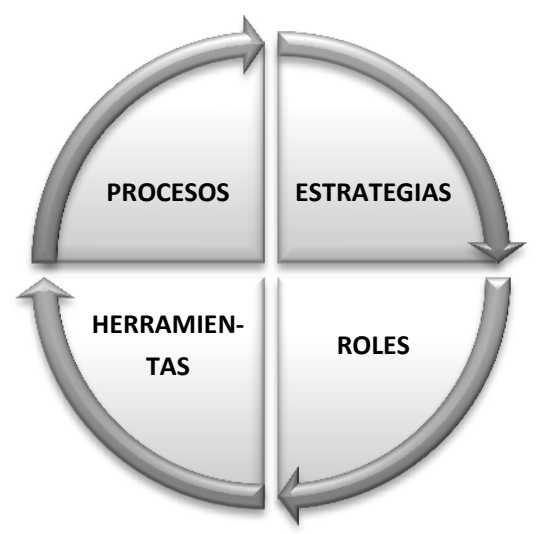

Fig. 2. Modelo PERH

\section{CONCLUSIONES}

Uno de los principales activos de la Gestión de Comunicaciones, es el fomento de valores de comunicación clara y abierta (Morales, F., 2001). En los proyectos de telecomunicaciones no existe 
cultura de gestión, una causa posible de esta situación radica en que los ingenieros en particular, han sido formados con un enfoque fuerte en lo técnico y pobre en lo relacionado a la gestión. Ello lo ratifica el consultor en comunicación (Bustamante, A., 2010) cuando manifiesta que de todos modos no resulta fácil hacer entender a los líderes del proyecto que desde siempre han vivido pendientes de las finanzas que las herramientas de la comunicación son el único denominador común a todos los actos y propósitos de su organización.

En particular, los proyectos de telecomunicaciones no pueden seguir siendo ajenos a la implementación de mecanismos de gestión en todas sus fases de desarrollo sin importar la envergadura del mismo. La correcta gestión de la comunicación dentro de las organizaciones reduce la posibilidad de los flujos incontrolados de información, disminuyendo así en un alto grado el riesgo de conflicto. Por ello, esta revisión brindó herramientas bases para que a partir de ella se consolidara un modelo de Gestión de Comunicaciones. El modelo planteado para la gestión de comunicaciones, se estructuró de acuerdo a las características y necesidades de los proyectos desarrollados en telecomunicaciones, ello basado en la experiencia de los autores y con la ayuda de la información recolectada. Los detalles del modelo PERH no están dentro de los alcances de este documento.

\section{REFERENCIAS}

Archibald, R., What CEO's must demand to achieve effective Project Management, Iberoamerican, Project management Forum, México. 2000.

Álvarez, A., y Lesta, L., Measuring the Contribution of Strategic Management of In-House Communication to the Objectives of the Organization, Revista Palabra Clave, Vol. 14, No 1, 2011, pp. 11-30

Álvarez, A., Medición y evaluación en comunicación, Instituto de Investigación en Relaciones Públicas (IIRP), 2011, pp 34. España.

Arce, L., y López, S., Considerations about Company Project Management in Bogota, Colombia- maturity level of project management, Revista Escuela de Administración de Negocios. Universidad EAN. Bogotá, Colombia, 2010, Pp. 60-87. 
Bustos, M., Cuevas, A., y Panqueva, M., Un caso de gestión de la comunicación y la información en la pyme manufacturera de calzado chiquitines, Revista EAN. Universidad EAN, Bogotá, Colombia, 2010.

Bustamante, A., Comunicaciones Cortadas A Medida, Red DirCom Iberoamericana. Recuperado de http://www.reddircom.org/textos/comunicaciones_medida.pdf.

Ballesteros, D., Frederick, T., y su Teoría Científica, Universidad Nacional de Colombia, Sede Mani-zales, Caldas-Colombia, 2010.

Butler, A., La administración de proyectos sus funciones sus errores. En Cleland \& King. Manual para la administración de proyectos, Editorial CECSA. Mexico, 1990, pp 75

Clemenza, C., Ferrer, J., Romero, D., y Araujo, R., Fortalecimiento de la Comunicación Organizacional, Revista Encuentro Educacional. Vol. 9 $\mathrm{N}^{\circ}$ 2. Universidad del Zulia, Venezuela, 2002.

Conalep. (2013). Definición y ejemplos de Proyecto, Educación de Calidad para la Competitividad. Estado de Querétano. México. Recuperado de http://tutoriales.conalepqro.edu.mx/lalo/Templates/unouno.html

Cornelissen, J., Corporate communication, London: Sage. 2008.

Estepa, R., (2004). Evolución Histórica de las Telecomunicaciones, Notas de ARSS. Recuperado de http://trajano.us.es/ rafa/ARSS/apuntes/tema1.pdf.

Freeman, C., y Louca, F., As time goes by: from the industrial revolution to the information, Oxford, Oxford, University Press, 2001.

Fernández, C., La Comunicación en las Organizaciones, Editorial Trillas, México, 1991.

Franta, W., (2001) LAN inalábrica- Aspectos radioeléctricos, Universidad de Rioja, España. Recuperado de http://uem.es/binaria/monograficos/archivos_monograficos/wolfgang_f ranta_espanol.pdf.

Gibson, J., Las Organizacione. Componente y Estructura de Procesos, Editorial Mc Graw Hill. 8va edición, Chile, 1999.

Goldhaber, G., La Comunicación Organizacional, Pera Edición. Editorial Diana, México, 1984.

Grunig, E., Symmetrical systems of internal communication, En Grunig, E. (ed.). Excellence in public relations andcommunication management. New Jersey: Lawrence Erlbaum Associates, EE.UU, 1992.

Gyepro., (2005). Breve Reseña Teórica de la Gestión de Proyectos, Universidad del Valle, Co-lombia. Recuperado de http://gyepro.univalle.edu.co/documentos/breve_resena_teorica_gp.pdf

Garrido, F. J., Comunicación, estrategia y empresa. Asociación Iberoamericana de Comunica-ción Estratégica, Medellín: Editorial Zuluaga, Colombia, 2003. 
Grunig, E., Prefacio a la edición en castellano, En Grunig, J. E. y Hunt, T. Dirección de relaciones públicas. Edición adaptada por Jordi Xifra, Barcelona: Gestión, 2000.

Joskowicz, J., Historia de las Telecomunicaciones, Instituto de Ingeniería Eléctrica, Facultad de Ingeniería, Universidad de la República Montevideo, Uruguay, 2012.

Microsoft., (2012) Historia breve de la administración de proyectos. Recuperado de http://office.microsoft.com

Morales, F., La Comunicación Interna. Herramienta estratégica de gestión para las empresas, Universidad Autónoma de Barcelona, 2001.

Manucci, M., La estrategia de los cuatro círculos, Editorial Norma, Bogotá Colombia, 2006.

Miranda, E., y Carvalho, A., Publicaciones Póstumas de Henry Fayol: Revisando su Teoría Ad-ministrativa, Universidad Previteriana, Brasil. Revista de Administración, Vol 12, 2011, pp 204-227.

Morales, R., y González, V., Comunicación en las Organizaciones y TIC: Un Estudio de Caso, Universidad de La Frontera, Francisco Salazar, Temuco, Chile, 2010.

Palacio, J., (2006). Origen de la gestión de proyectos. Recuperado de http://www.navegapolis.net.

PMBOK., Una Guía a los Fundamentos de la Dirección de Proyectos, PMBOK Guide, Cuarta Edición PMI. Project Management Institute. 2008.

Petrella, C. Análisis de la Teoría Burocrática, Revista Electrónica de la Facultad de Ciencias Empresariales, Universidad Católica del Uruguay, 2007.

Pérez, R. A., Estrategias de comunicación, Barcelona: Editorial Ariel. 2006.

Preciado A. y Ramírez H., Gestión de la comunicación estratégica en los sectores empresarial, de desarrollo y público, Revista Palabra Clave, Universidad de La Sabana, Colombia, 2012.

Petit, A., El plan de comunicación: génesis y desarrollo, En Bel Mallén (coord.), Comunicar para crear valor. La dirección de comunicación en las organizaciones, Pamplona: Eunsa, 2004, (pp. 171-196).

Pizzolante, I. (2004). El poder de la comunicación estratégica, Bogotá: Ediciones Pontificia Universidad Javeriana, 2004.

Prieto, y Suárez., Perfil de las motivaciones sociales y clima organizacional. Caso: personal administrativo, Revista de Ciencias Sociales. Vol 4 $\mathrm{N}^{\circ}$ 1. Universidad del Zulia, Maracaibo, Venezuela. 1998.

Quintín., Retórica, Introducción, traducción y notas por Quintín. Racionero, Madrid: Gredos. 1990. 
Rivera, A. B., Rojas, L. R., Ramírez, F. and Álvarez F., T., Communication as Organizational Management Tool, Revista NEGOTIUM Ciencias Gerenciales Año. 2005, 1/N² 2 /2005, pp 32-48.

Ramirez, F., y Rojas, L., Desarrollo de la Identidad Corporativa: Función Inherente a la Ges-tión Comunicacional, Revista Multiciencia, Vol 4. $\mathrm{N}^{\circ}$ 1. Punto Fijo, Venezuela, 2004.

Rojas, L., y Arape, E., La Visión y la Comunicación en Gerencia, Revista Opción. Vol 15 N²8, Maracaibo, Venezuela, 1999.

Senge, P., La Quinta Disciplina, ediciones Juan Gringa S.A. España, 1995.

Serna, H., La Gestión Empresarial, Editorial Legis. Fondo Editorial, Colombia, 2000.

Sotelo, C., Introducción a la comunicación institucional, Barcelona: Ariel, 2001.

Srivannaboon, S., Linking Project Management with Business Strategy, Project Management Journal, Volumen 37, Diciembre, 2005, pág. 88.

Scheinsohn, D., Strategic Communication. Management and corporate fundamentals, Ediciones Macchi, 1993.

Timm, P., Managerial Comunication: a finger on the pulse, Prentice Hall: Englewood Cliffs, 1986.

Ugas, L., The management of the projects in the companies of the power sector. Case: ENELVEN - CARBOZULIA, Universidad Rafael Belloso, Chacín, Venezuela, 2008, Pp. 70-97.

Van, C., Comunicación Corporativa, Editorial Prentice Hill. España, 1997.

Vargas, L., Tesis: Gestión Comunicacional e IdentidadCorporativa de la Organización Universitaria, Facultad de Humanidades y Educación, Universidad del Zulia, Venezuela, 2003.

Watzlawick, P., Teoría de la comunicación humana, España: Herder, 1981. 